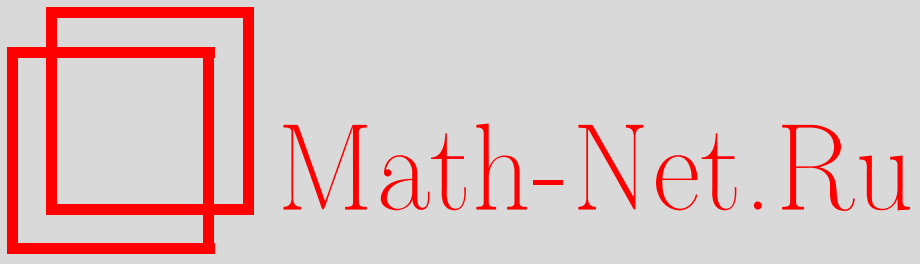

И. А. Ибрагимов, Об оценке многомерной аналитической плотности распределения по цензурированной выборке, Теория вероятн. и ее примен., 2006, том 51, выпуск 1, 95-108

DOI: https://doi.org/10.4213/tvp148

Использование Общероссийского математического портала MathNet.Ru подразумевает, что вы прочитали и согласны с пользовательским соглашением

http://www.mathnet.ru/rus/agreement

Параметры загрузки:

IP : 54.81 .137 .203

26 апреля 2023 г., 18:24:51

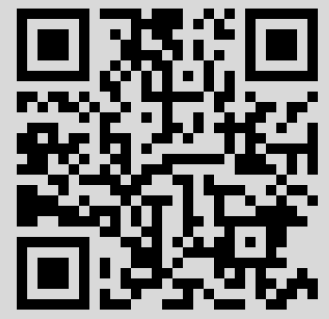




\section{ОБ ОЦЕНКЕ МНОГОМЕРНОЙ АНАЛИТИЧЕСКОЙ ПЛОТНОСТИ РАСПРЕДЕЛЕНИЯ ПО ЦЕНЗУРИРОВАННОЙ ВЫБОРКЕ ${ }^{1)}$}

Изучается задача оценивания неизвестной плотности распределения $f(x)$ по $d$-мерной выборке $X_{1}, \ldots, X_{n}, X_{j} \in \mathbf{R}^{d}$, в предположении, что наблюдаются лишь те элементы выборки, которые попадают в заданную ограниченную область $G \subset \mathbf{R}^{d}$.

Ключевые слова и фразы: плотность распределения, непараметрические оценки, аналитические функции, проективные оценки.

1. Постановка задачи. Результаты. Пусть

$$
X_{1}, X_{2}, \ldots, X_{n}
$$

есть выборка из $d$-мерного распределения с неизвестной плотностью распределения $f(x)=f\left(x_{1}, \ldots, x_{d}\right)$. Мы предполагаем, что $f \in \mathbf{F}$, где $\mathbf{F}-$ некоторый известный класс функций. Задача заключается в оценивании $f$ по выборке (1.1) в предположении, что наблюдателю доступны лишь те из наблюдений (1.1), которые попадают в область $G$. Наблюдения, не попавшие в $G$, не регистрируются и не могут быть использованы для построения оценки. Номера реально наблюдавшихся $X_{j}$ не предполагаются известными. Общее количество наблюдений $n$, как зарегистрированньх, так и незарегистрированных, предполагается известным.

Можно ожидать, и это действительно так, что определенное выше цензурирование не позволяет состоятельно оценить $f(x)$ для $x \notin G$, даже если F - класс бесконечно дифференцируемых функций (достаточно рассмотреть две бесконечно дифференцируемые плотности распределения, совпадаюшие на $G$ и различаюшихся вне некоторой окрестности $G$ ). С другой стороны, цензурирование не очень сильно влияет на точность оценивания $f(x)$, когда $x \in G$, если $\mathbf{F}$ - класс бесконечно дифференцируемых функций.

* С.-Петербургское отделение Математического института им. В. А. Стеклова РАН, математико-механический факультет СПбГУ С.-Петербург, Россия; е-mail: ibr32@pdmi.ras.ru

1) Работа выполнена при частичной поддержке РФФИ, грант 05-01-00920, и РФФИDFG, грант 04-01-04000. 
Аналитическая в комплексной области $\widehat{G} \subset \mathbf{C}^{d}, G \subset \widehat{G}$, функция $f(z)=f\left(z_{1}, \ldots, z_{d}\right)$ однозначно определяется во всей области $\widehat{G}$ своими значениями в вещественной области $G$ и потому можно ожидать, вопервых, что по наблюдениям цензурированным областью $G$ целую аналитическую плотность распределения $f(x)$ возможно состоятельно оценить в более широкой $R_{n}$-окрестности области $G\left(R_{n} \rightarrow \infty, n \rightarrow \infty\right)$; и, во-вторых, что потеря наблюдений $X_{j} \notin G$ существенно снижает точность оценивания целой аналитической плотности $f(x)$ внутри множества $G$. Для $d=1$ эти задачи рассмотрены в работе автора [4]; ниже исследуется случай $d>1$.

Ответ на первый вопрос дают формулируемые ниже теоремы 1.1, 1.2 (ср. [8, теоремы $1.1,1.2]$ и [4]). Обозначим $\mathbf{F}=\mathbf{F}_{d}(M, \sigma, \rho)$, где $\sigma=\left(\sigma_{1}, \ldots, \sigma_{d}\right), \rho=\left(\rho_{1}, \ldots, \rho_{d}\right)$, класс целых аналитических плотностей распределения таких, что

$$
\sup _{\left|z_{i}\right| \leqslant R_{i}, i=1, \ldots, d}|f(z)| \leqslant M \exp \left\{\sum_{1}^{d} \sigma_{i} R_{i}^{\rho_{i}}\right\} .
$$

Здесь $M>0, \sigma_{i}>0, \rho_{i} \geqslant 1$ - параметры, определяюшие класс. Зависимость от размерности $d$ ниже, как правило, не указывается. Ограничение $\rho_{i} \geqslant 1$ объясняется тем, что целых аналитических плотностей распределения с $\min _{i} \rho_{i}<1$ не существует. (Действительно, пусть, например, $\rho_{1}<1$. При фиксированных $x_{2}, \ldots, x_{d}$ функция $F(z)=$ $\int_{0}^{z} f\left(u, x_{2}, \ldots, x_{d}\right) d u$ есть целая аналитическая функция $z$ порядка $\rho_{1}<1$, ограниченная по модулю единицей на вещественной оси. В силу принципа Фрагмена-Линделефа $F(z)$ ограничена в верхней и нижней полуплоскостях и, следовательно, является константой.)

Теорема 1.1. Пусть $\mathbf{F}=\mathbf{F}(M, \sigma, \rho)$. Обозначим $G_{n}(\alpha) R_{n}$-охрестность множества $G$, аде $R_{n}=R_{n}(\alpha)=(\ln n)^{\alpha / e}$. Здесь $\varrho=\max \rho_{i} u$ $0<\alpha<1$. Существует такая оченка $\hat{f}(x)$ плотности $f(x)$, построенная по чензурированным наблюдениям (1.1), ито для любых фиксированных $\alpha$ и $\beta, \beta<\alpha<1$, выполндется неравенство

$$
\sup _{f \in \mathbf{F}} \mathbf{E}_{f}\left\{\sup _{G_{\beta}}|f(x)-\hat{f}(x)|\right\} \leqslant C_{\alpha, \beta} n^{-(1-\alpha) /(2 d)} .
$$

Постояннье $C_{\alpha, \beta}$ зависят тажже от $M, \sigma, \rho, d, G$.

Следуюшая ниже теорема показывает, что размер области состоятельности $G_{n}$ предыдущей теоремы не может быть существенно увеличен.

Теорема 1.2. Пусть $\mathbf{F}=\mathbf{F}(M, \sigma, \rho)$. Пусть $x-$ произвольная точка внешности множества $G_{n}(\alpha), \alpha>1$. Тогда для всех $n \geqslant n_{0}(\alpha, M, \sigma, \rho)$

$$
\inf _{\hat{f}} \sup _{f \in \mathbf{F}} \mathbf{E}_{f}|f(x)-\hat{f}|^{2}>c_{0},
$$

$c_{0}$ зависит от $\alpha, M, \sigma, \rho$. 
При ответе на второй вопрос ограничимся плотностями класса $\mathbf{F}(M, \sigma, \mathbf{1})$, т.е. плотностями, которые являются целыми функциями экспоненциального типа. В этом случае функция $f \in L_{2}\left(\mathbf{R}^{d}\right)$ и по теореме Пэли-Винера она допускает представление вида (см., например, [11])

$$
f(x)=\int_{K} e^{-i(t, x)} \psi(t) d t, \quad \psi \in L_{2},
$$

где $K$ - ограниченное симметричное выпуклое тело в $\mathbf{R}^{d}(\psi(t)$, разумеется, характеристическая функция элементов выборки). Обозначим класс плотностей распределения, допускаюших представление (1.5) через $\mathbf{F}(K)$. В работе [7] было доказано, что при оценивание плотностей класса $\mathbf{F}(K)$ по нецензурированной выборке (1.1) справедливо соотношение

$$
\inf _{\hat{f}} \sup _{f \in \mathbf{F}_{\sigma}} \mathbf{E}_{f}\|f-\hat{f}\|_{2}^{2} \sim \frac{1}{(2 \pi)^{d}} \cdot \frac{1}{n}(1+o(1)), \quad n \rightarrow \infty .
$$

Следуюшая теорема показывает, как меняется скорость сходимости в (1.6) при переходе к цензурированным выборкам.

Теорема 1.3. В задаче оченивания плотностей $f$ класса $\mathbf{F}(K)$ по чензурированной областью $G$ выборке (1.1)

$$
\inf _{\hat{f}} \sup _{f \in \mathbf{F}(K)} \mathbf{E}_{f} \int_{G}|f(x)-\hat{f}(x)|^{2} d x \asymp \frac{1}{n}\left(\frac{\ln n}{\ln \ln n}\right)^{d} .
$$

Порядок убывания правых частей в сформулированных выше теоремах не зависит от $G$. Поэтому естественно при доказательстве этих теорем выбрать в качестве $G$ достаточно простые множества, скажем, кубы. Если теоремы будут доказаны для всех кубов, то в силу того, что для любой ограниченной области $G$ найдутся кубы $\Gamma_{1}, \Gamma_{2}$ такие, что $\Gamma_{1} \in G \in \Gamma_{2}$, теоремы будут доказаны для всех $G$. При этом ясно, что достаточно рассмотреть какой-нибудь один куб. Поэтому ниже в ходе доказательства мы всегда предполагаем, что $G=[-1,1]^{d}$.

2. Верхние границы. Конструкция оценок. Доказательство теоремы 1.1. Ниже всюду рассматривается следуюшая единообразная схема построения «хороших» оценок (проекционные оценки Н. Н. Ченцова [14]). Именно, выбирается подходящее разложение

$$
f(x)=\sum_{j} a_{j} \phi_{j}(x)
$$

по базисным аналитическим функциям $\phi_{j}$, коэффициенты $a_{j}$ оцениваются оценками $\widehat{a}_{j}$ и в качестве оценок для $f$ рассматриваются статистики

$$
\hat{f}(x)=\sum_{0}^{N} \widehat{a}_{j} \phi_{j}(x)
$$


причем выбор $N$ зависит от априорных сведений $\mathbf{F}$ о плотности $f(x)$ (см. ниже, окончание доказательства теоремы).

В силу наших предположений относительно $G, G=[-1,1]^{d}$, в качестве функций $\phi_{j}$ естественно выбрать классические полиномы Лежандра $P_{j}(x)$ (точнее их многомерные аналоги), свойства которых хорошо изучены (см., например, [10]). Именно, для $x=\left(x_{1}, \ldots, x_{d}\right)$, $k=\left(k_{1}, \ldots, k_{d}\right)$ положим $P_{k}(x)=P_{k_{1}}\left(x_{1}\right) \cdots P_{k_{d}}\left(x_{d}\right)$, где $P_{k_{j}}\left(x_{j}\right)-$ opтонормированные на $[-1,1]$ полиномы Лежандра. Полиномы Лежандpa $P_{j}(y)$ образуют ортонормальный базис в $L_{2}(-1,1)$ и потому многомерные полиномы Лежандра образуют ортонормальный базис в $L_{2}(G)$. В частности, сужение плотности $f$ на $G$ разлагается в ряд Фурье по полиномам Лежандра,

$$
f(x)=\sum_{k} a_{k} P_{k}(x),
$$

причем коэффициенты Фурье-Лежандра

$$
a_{k}=\int_{G} f(x) P_{k}(x) d x .
$$

Условимся ниже посредством $c$ и $C$ с индексами или без обозначать константы (не обязательно одни и те же даже при полном графическом совпадении), зависяшие лишь от $M, \sigma, \rho, d$.

Лемма 2.1. Если $f \in \mathbf{F}(M, \sigma, \rho)$, mo

$$
\left|a_{k}\right| \leqslant c M \exp \left\{-\frac{k_{1}}{\rho_{1}} \ln \frac{k_{1}}{e \rho_{1} \sigma_{1}}-\cdots-\frac{k_{d}}{\rho_{d}} \ln \frac{k_{d}}{e \rho_{d} \sigma_{d}}\right\} .
$$

Д о к а з а т е л с с в о. По формуле Родрига для ортонормированных полиномов Лежандра ([10, с. 79$])$

$$
P_{j}(y)=\sqrt{\frac{2 j+1}{2}} \frac{(-1)^{j}}{2^{j} j !} \frac{d^{j}}{d y^{j}}\left\{\left(1-y^{2}\right)^{j}\right\} .
$$

Подставляя последнее соотношение в (2.2) и интегрируя по частям, мы найдем, что艹

$$
a_{k}=\prod_{j=1}^{d} \sqrt{\frac{2 k_{j}+1}{2}} \frac{1}{k_{j} !} \cdot \int_{G} D^{k} f(x) \prod_{j=1}^{d}\left(1-x_{j}^{2}\right)^{k_{j}} d x_{1} \cdots d x_{d},
$$

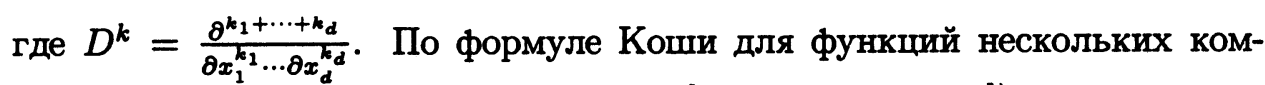
плексных переменных (см., например, [13, теорема 2.2.1])

$$
D^{k} f(x)=\frac{k_{1} ! \cdots k_{d} !}{(2 i \pi)^{d}} \int_{\Gamma} \frac{(-1)^{k_{1}+\cdots+k_{d}} f(\zeta)}{\left(\zeta_{1}-x_{1}\right)^{k_{1}+1} \cdots\left(\zeta_{d}-x_{d}\right)^{k_{d}+1}} d \zeta_{1} \cdots d \zeta_{d}
$$


интегрирование ведется по множеству $\Gamma=\left\{z:\left|z_{j}\right|=R_{j}, j=1, \ldots, d\right\}$, $R_{j}>1$. Из последнего равенства следует, что для всех $f \in \mathbf{F}(M, \sigma, \rho)$, $x \in G$ и $R_{j} \geqslant 2$

$$
\left|D^{k} f(x)\right| \leqslant M 2^{d} \prod_{j=1}^{d} 2^{k_{j}} R_{j}^{-k_{j}} \exp \left\{\sigma_{j} R_{j}^{\rho_{j}}\right\} .
$$

Подставляя последнее неравенство в (2.4) и минимизируя по $R_{j}$, мы получим (2.3). Лемма доказана.

Лемма 2.2 (см. [10]). Имеют место неравенства:

$$
\max _{|y| \leqslant 1}\left|P_{j}(y)\right| \leqslant\left|P_{j}(1)\right|=\sqrt{\frac{2 j+1}{2}} .
$$

Лемма 2.3 (см. [1, с. 74]). Если $Q(z), z \in \mathbf{C}^{1}$, - полином степени $n$, то

$$
|Q(z)| \leqslant \max _{|x| \leqslant 1}|Q(x)| \cdot\left|z+\sqrt{z^{2}-1}\right|^{n} .
$$

Из трех последних лемм следует, что ряд в правой части (2.1) сходится для всех $z \in \mathbf{C}^{d}$ к некоторой целой аналитической функции; в $G$ этот ряд сходится к $f(x)$ и, следовательно, для всех $z \in \mathbf{C}^{d}$

$$
f(z)=\sum_{k} a_{k} P_{k}(z)
$$

Определим статистики $f_{N}(x)$ равенствами

$$
f_{N}(x)=\sum_{k_{j} \leqslant N} \widehat{a}_{k} P_{k}(x)
$$

где оценки $\widehat{a}_{k}$ коэффициентов $a_{k}$ в свою очередь определяются соотношениями $\widehat{a}_{k}=n^{-1} \sum_{j: X_{j} \in G} P_{k}\left(X_{j}\right)=n^{-1} \sum_{j=1}^{n} P_{k}\left(X_{j}\right) I\left(X_{j}\right)$. Здесь и ниже $I(x)$ обозначает индикатор множества $G$. Статистики $\widehat{a}_{k}$ суть несмещенные оценки для $a_{k}$, Е $\widehat{a}_{k}=\int_{G} P_{k}(x) f(x) d x=a_{k}$. (Математическое ожидание вычисляется по отношению к «истинной» плотности распределения $f$ и правильнее было бы писать $\mathbf{E}_{f}(\cdot)$. Но мы, как правило, опускаем индекс $f$, сохраняя его лишь в тех случаях, когда его отсутствие может привести к недоразумению.) Отсюда и из леммы 2.1 следует, что

$$
\int_{G}\left|f(x)-\mathbf{E} f_{N}(x)\right|^{2} d x=\sum_{j=1}^{d} \sum_{k: k_{j}>N}\left|a_{k}\right|^{2} \leqslant C \sum_{j=1}^{d} \exp \left\{-\frac{N}{2 \rho_{j}} \ln \frac{N}{\rho_{j}}\right\} \text {. }
$$

Из лемм 2.1, 2.2 вытекает, что $\sup _{f \in \mathbf{F}}\|f\|_{L_{\infty}(G)}=C<\infty$. Поэтому

$$
\begin{aligned}
& \mathbf{E}\left\{\int_{G}\left|f_{N}(x)-\mathbf{E} f_{N}(x)\right|^{2} d x\right\}=\sum_{k_{i} \leqslant N} \mathbf{E}\left|\widehat{a}_{k}-a_{k}\right|^{2} \\
& \leqslant \frac{1}{n} \sum_{k_{i} \leqslant N} \int_{G} P_{k}^{2}(x) f(x) d x \leqslant C \frac{N^{d}}{n} .
\end{aligned}
$$


Отсюда и из (2.9) следует, что

$$
\mathbf{E} \int_{G}\left|f(x)-f_{N}(x)\right|^{2} d x \leqslant C\left(\frac{N^{d}}{n}+\sum_{1}^{d} \exp \left\{-\frac{N}{\rho_{j}} \ln \frac{N}{\rho_{j}}\right\}\right) .
$$

Выбирая $N \sim 2\left(\max _{j} \rho_{j}\right) \ln n / \ln \ln n$, и полагая $\hat{f}(x)=f_{N}(x)$, приходим к следуюшему результату, даюшему нужную верхнюю границу в (1.7).

Теорема 2.1. Пусть $\mathbf{F}=\mathbf{F}(M, \sigma, \rho)$. Существует такая оченка $\hat{f}(x)$ плотности $f(x)$ по иензурированной выборке (1.1), что

$$
\sup _{f \in \mathbf{F}} \mathbf{E}_{f} \int_{G}|f(x)-\hat{f}(x)|^{2} d x \leqslant C \frac{1}{n}\left(\frac{\ln n}{\ln \ln n}\right)^{d} .
$$

Чтобы доказать неравенство теоремы 1.1 , заметим сперва, что в силу лемм 2.1-2.3

$$
\begin{aligned}
& \sup _{\left|x_{i}\right| \leqslant R}\left|f(x)-\mathbf{E} f_{N}(x)\right| \leqslant C \prod_{i=1}^{d} \sum_{j=0}^{\infty} R^{j} \exp \left\{-\frac{j}{\rho_{i}} \ln \left(\frac{j}{e \sigma_{i} \rho_{i}}\right)\right\} \\
& \quad \times \sum_{l=1}^{d} \sum_{N+1}^{\infty} R^{j} \exp \left\{-\frac{j}{\rho_{i}} \ln \left(\frac{j}{e \sigma_{i} \rho_{i}}\right)\right\} \\
& \quad \leqslant C R^{N} \exp \left\{c\left(R^{\rho_{1}}+\cdots+R^{\rho_{d}}\right)\right\} \exp \left\{-\frac{N}{\varrho} \ln \frac{N}{\varrho}(1+o(1))\right\} .
\end{aligned}
$$

Поэтому для таких $R$, что $\ln R<[(1-t) / \varrho] \ln N$, где $t>0$,

$$
\sup _{\left|x_{i}\right| \leqslant R}\left|f(x)-\mathbf{E} f_{N}(x)\right| \leqslant C_{t} R^{N} e^{-(N / \rho) \ln (N / \ell)} .
$$

Далее, снова на основании лемм 2.1-2.3

$$
\begin{gathered}
\mathbf{E} \sup _{|x| \leqslant R}\left|f_{N}(x)-\mathbf{E} f_{N}(x)\right| \leqslant \sum_{k_{i} \leqslant N} R^{k_{1}} \cdots R^{k_{d}} \mathbf{E}\left|\widehat{a}_{k}-a_{k}\right| \\
\leqslant C R^{N d} \sum_{k_{i} \leqslant N} \int_{G}\left(\left|P_{k}(x)\right|^{2} f(x) d x\right)^{1 / 2} \leqslant C \frac{N^{d} R^{d N}}{\sqrt{n}} .
\end{gathered}
$$

Из (2.14) и (2.15) следует, что

$$
\begin{aligned}
\mathbf{E} \sup _{\left|x_{i}\right| \leqslant R}\left|f(x)-f_{N}(x)\right| \leqslant C_{t} & \left(\exp \left\{\frac{(1-t) d}{\varrho} N \ln N\right\} N^{d} n^{-1 / 2}\right. \\
& \left.+\exp \left\{-\frac{t N}{\varrho} \ln N\right\}\right) .
\end{aligned}
$$

Полагая здесь $N \sim c \ln n / \ln \ln n$ и подбирая $c$ должным образом, придем к неравенству (1.3). Теорема 1.1 доказана. 
3. Доказательство теоремы 1.2. Мы докажем теорему 1.2 для случая $\rho>1$. Случай $\rho=1$ по существу разобран в работе [4]. Кроме того, для простоты мы не будем доказывать теорему для класса $\mathbf{F}(M, \sigma, \rho)$ с заданными $M, \sigma$, а проведем доказательство для класса $\mathbf{F}(M, \sigma, \rho)$ с заданным $\rho$ и какими-то $M$ и $\sigma$. Конструкцию доказательства легко дополнить так, чтобы рассмотреть заданные $M, \sigma$.

Изложим общую схему доказательства теоремы 1.2 . Без потери обшности можно предположить, что оценивается значение плотности $f(x)$ в точке $x^{T}=(T, 0, \ldots, 0), T>0$. Рассмотрим однопараметрическое, зависящее от параметра $\theta \in[-\delta, \delta]$, семейство плотностей распределения $f(x, \theta)$ вида

$$
\begin{aligned}
f(x, \theta)=f_{T}(x, \theta)= & \frac{1}{2}\left(\varphi\left(x-x^{T}\right)+\varphi\left(x+x^{T}\right)\right) \\
& \times\left(1+\theta \sin \left(\frac{\pi x_{1}}{2 T}\right)\left(g\left(x-x^{T}\right)+g\left(x+x^{T}\right)\right)\right) .
\end{aligned}
$$

Здесь $\varphi(x)=\prod_{1}^{d} \sin ^{2} x_{i} /\left(\pi x_{i}^{2}\right)$ есть плотность распределения класса $\mathbf{F}(M, \sigma, \rho), \rho=(1, \ldots, 1)$, а $g(x)$ - это четная равномерно ограниченная на всей вешественной гиперплоскости функция класса $\mathbf{F}(M, \sigma, \rho)$, $\rho=\left(\rho_{1}, \ldots, \rho_{d}\right), \rho_{1}=\max _{i} \rho_{i}=\varrho>1$. Функции $f(x, \theta)$ - действительно плотности распределения, во всяком случае для достаточно малых $\delta$. Действительно, в силу ограниченности $g$ для малых $\delta$ выполняется неравенство $f(x, \theta) \geqslant 0$, кроме того, в силу четности функции $g$

$$
\int_{\mathbf{R}^{d}} f(x, \theta) d x=\int_{\mathbf{R}^{d}} \varphi(x) d x=1 .
$$

При всех значениях параметра $\theta \in[-\delta, \delta]$ плотности $f(x, \theta) \in \mathbf{F}$ и

$$
\inf _{\hat{f}} \sup _{f \in \mathbf{F}}\left|f\left(x^{T}\right)-\hat{f}\right| \geqslant \inf _{\hat{f}} \sup _{\theta \in[-\delta, \delta]}\left|f\left(x^{T}, \theta\right)-\hat{f}\right| .
$$

Рассмотрим теперь наряду с нашей основной задачей следующую первую вспомогательную задачу оценивания: оценить параметр $\theta$ по цензурированной выборке (1.1) с плотностью распределения элементов выборки равной $f(x ; \theta)$ из семейства (3.1) (при этом мы, как и выше, предполагаем, что цензурирующее множество $\left.G=[-1,1]^{d}\right)$. Если $\hat{f}-$ какая-нибудь оценка значения $f\left(x^{T}\right)$ плотности $f(x)$ в основной задаче, то статистика

$$
\hat{\theta}=\frac{2 \hat{f}-\left(\varphi(0)+\varphi\left(2 x^{T}\right)\right)}{g(0)+g\left(2 x^{T}\right)}
$$

может быть принята в качестве оценки параметра $\theta$ первой вспомогательной задачи. При этом

$$
\mathbf{E}_{f(; ; \theta)}|\hat{\theta}-\theta|^{2}=2 \frac{\mathbf{F}_{f}|\hat{f}-f|^{2}}{g(0)+g\left(2 x^{T}\right)}
$$


Если, поэтому, $g$ выбрано так, что

$$
g(0)=1, \quad\left|g\left(2 x^{T}\right)\right| \leqslant \frac{1}{2}
$$

то $\mathbf{E}|\hat{\theta}-\theta|^{2} \leqslant 4 \mathbf{E}\left|\hat{f}-f\left(x^{T}, \theta\right)\right|^{2}$ и, потому,

$$
\inf _{\hat{f}} \sup _{f \in \mathbf{F}} \mathbf{E}_{f}\left|f\left(x^{T}\right)-\hat{f}\right|^{2} \geqslant 4^{-1} \inf _{\hat{\theta}} \sup _{\theta \in[-\delta, \delta]} \mathbf{E}_{\theta}|\theta-\hat{\theta}|^{2},
$$

причем слева нижняя грань берется по всем оценкам $\hat{f}$ основной задачи, а справа по всем оценкам $\hat{\theta}$ первой вспомогательной задачи. Таким образом, чтобы доказать теорему 1.2, нам достаточно показать, что для всех больших $n$ правая часть в (3.5) больше положительной постоянной.

Введем в рассмотрение вторую вспомогательную задачу оценивания: оценить параметр $\theta$ по наблюдениям

$$
Y_{1}, Y_{2}, \ldots, Y_{n},
$$

где $Y_{j}=X_{j} I\left(X_{j}\right)$, а $I(x)$, как и выше, означает индикатор множества $G$. Если $\theta^{*}$ пробегает класс $\Theta^{*}$ всех статистик $\theta^{*}\left(Y_{1}, \ldots, Y_{n}\right)$, а $\hat{\theta}-$ класс $\widehat{\Theta}$ всех статистик, построенных по цензурированной выборке, то, очевидно, $\widehat{\Theta} \subseteq \Theta^{*}$ и, потому,

$$
\inf _{\Theta^{*}} \sup _{\theta} \mathbf{E}_{\theta}\left|\theta^{*}-\theta\right|^{2} \leqslant \inf _{\widehat{\Theta}} \sup _{\theta} \mathbf{E}_{\theta}|\hat{\theta}-\theta|^{2} .
$$

Из (3.6) и (3.7) вытекает, что неравенство (1.4) теоремы 1.2 следует из неравенства: для всех достаточно больших $n$

$$
\inf _{\Theta^{*}} \sup _{\theta} \mathbf{E}_{\theta}\left|\theta^{*}-\theta\right|^{2} \geqslant c_{1}>0 .
$$

Это последнее неравенство мы и будем доказывать.

Лемма 3.1. Рассмотрим задачу оченивания параметра $\theta \in[-\delta, \delta]$ по выборке $Y_{1}, \ldots, Y_{n}$ из распределения с плотностью $f(x, \theta)$ и информачией Фишера $I(\theta)$. Если для всех $\theta \in[-\delta, \delta]$ информачия Фишера $I(\theta) \leqslant n^{-1}$, mo

$$
\inf _{\hat{\theta}} \sup _{\theta} \mathbf{E}_{\theta}|\hat{\theta}-\theta|^{2} \geqslant c(\delta), \quad c(\delta)>0 .
$$

Неравенство леммы легко следует из неравенства Крамера-Рао, см. подробнее [4].

Лемма 3.2. Информачия Фишера $I(\theta)$ о параметре $\theta$ во второй вспомогательной задаче равна

$$
\int_{G} \frac{\left[\varphi\left(x+x^{T}\right)+\varphi\left(x-x^{T}\right)\right] \sin ^{2}(\pi x / 2 T)\left[g\left(x-x^{T}\right)+g\left(x+x^{T}\right)\right]^{2}}{2\left(1+\theta \sin (\pi x / 2 T)\left(g\left(x-x^{T}\right)+g\left(x+x^{T}\right)\right)\right)} d x .
$$

Разъяснение этим вычислениям см в [4]. 
Теперь для окончания теоремы достаточно построить функции $g$ в (3.1) так, чтобы заданная выражением (3.10) информация Фишера удовлетворяла бы неравенствам (3.9). Мы построим функции $g(x)$ в виде

$$
g(x)=\gamma\left(x_{1}\right) \frac{\sin x_{2}}{x_{2}} \cdots \frac{\sin x_{d}}{x_{d}} .
$$

При этом для того, чтобы $g_{T}(x)=g\left(x-x^{T}\right) \in \mathbf{F}$, достаточно потребовать, чтобы $\gamma \in \mathbf{F}_{1}(M, \sigma, \varrho)$. Ниже при построении функции $\gamma$ точки $x$ и $z$ принадлежат соответственно $\mathbf{R}^{1}$ и $\mathbf{C}^{1}$. Мы рассмотрим по отдельности случаи, когда $\varrho$ - целое четное число, целое нечетное число, нецелое число.

1. Начнем с самого простого случая $-\varrho=2 k$ есть целое четное число. Положим $\gamma(x)=e^{-x^{2 k}}$ и убедимся, что при всех $T>0, \gamma_{T}=$ $\gamma(\cdot-T) \in \mathbf{F}_{1}$. Действительно, если $|z| \leqslant T / 2$, то $\left|\gamma_{T}(z)\right| \leqslant \exp \left\{-T^{2 k} / 2^{2 k}+\right.$ $\left.O\left(T^{2 k-2}\right)\right\} \leqslant M e^{-\sigma|z|^{\mathfrak{e}}}$, если же $|z|>T / 2$, то $|\gamma(z)| \leqslant e^{2^{e}|z|^{\mathfrak{e}}}$. Далее, для $|x| \leqslant 1$ справедливо $|\gamma(x-T)| \leqslant C e^{-c T^{e}}, c>0$, так что и $I(\theta) \leqslant C e^{-c T^{e}}$. Поэтому $I(\theta)<1 / n$ для $T>(2 / c) \ln n$ и всех достаточно больших $n$. Тем самым для целых четных $\varrho$ теорема доказана.

2. Пусть теперь $\varrho=2 k+1-$ это нечетное целое число. Положим

$$
\gamma(x)=\prod_{n=1}^{\infty} \frac{\sin x^{\ell} n^{-(1+\varepsilon)}}{x^{\ell} n^{-(1+\varepsilon)}}, \quad \varepsilon>0 .
$$

Для вещественных $x$ в силу формулы Стирлинга для факториалов

$$
|\gamma(x)| \leqslant \prod_{n<x^{e /(1+\varepsilon)}}\left(x^{e} n^{-(1+\varepsilon)}\right)^{-1} \leqslant C e^{-|x|^{e}} .
$$

Для $z=u+i v$

$$
\left|\frac{\sin z}{z}\right| \leqslant \frac{\left|e^{i z}\right|+\left|e^{-i z}\right|}{2|z|} \leqslant \frac{e^{|v|}}{|z|} .
$$

Поэтому аналогично (3.11) найдем, что $\left|\gamma_{T}(z)\right| \leqslant C e^{c|z|^{e}}$ для $|z| \leqslant T / 2$. Так как

$$
\left|\frac{\sin z}{z}\right|=\frac{1}{2}\left|\int_{-1}^{1} e^{i t z} d t\right| \leqslant e^{|z|}
$$

то $\left|\gamma_{T}(z)\right| \leqslant C e^{c|z|^{e}}$ для $|z|>T / 2$ и, следовательно, $\gamma \in \mathbf{F}_{1}$.

Из (3.11) следует, что информация Фишера $I(\theta) \leqslant C e^{-c T^{e}}$, а это, как мы уже видели, влечет неравенство теоремы 1.3.

3. Пусть теперь $p<\varrho<p+1, p \geqslant 1$ - целое. Обозначим $G(u, p)$ первичный множитель Вейерштрасса,

$$
G(u, p)=(1-u) \exp \left\{u+\frac{u^{2}}{2}+\cdots+\frac{u^{p}}{p}\right\} .
$$

Полагая $a_{r}=r^{1 / e}$, составим каноническое произведение рода $p$ (см. [9, гл. $1, \S 3])$

$$
V(z)=\prod_{1}^{\infty} G\left(\frac{z}{a_{r}}, p\right) .
$$


Каноническое произведение (3.12) равномерно сходится на всех компактных подмножествах $\mathbf{C}$, определяя тем самым целую аналитическую функцию $z$. Кроме того,

$$
\sup _{|z| \leqslant R}|V(z)| \leqslant c_{p} \exp \left\{\left((\varrho-p)^{-1}+(p+1-\varrho)^{-1}\right) R^{\varrho}\right\} .
$$

Доказательство этих хорошо известных фактов см., например, в монографии $[9, \S 3,4$ гл. 1$]$.

Лемма 3.3. Пусть $z=r e^{i \theta}, 0<\theta<2 \pi$. Тогда равномерно в любом интервале вида $0<\eta \leqslant \theta \leqslant 2 \pi-\eta$ при $r \rightarrow \infty$

$$
\ln V\left(r e^{i \theta}\right)=\frac{\pi}{\sin \pi \varrho} r^{\varrho} e^{i \varrho(\theta-\pi)}+O\left(r^{p}\right) .
$$

Мы опустим доказательство леммы, поскольку она является частным случаем теоремы 25 гл. I из [9], но с тем отличием, что в цитируемой теореме отсутствует оценка остаточного члена. Доказательство леммы повторяет доказательство цитируемой теоремы, а специальный вид последовательности $a_{r}, a_{r}=r^{1 / e}$, позволяет без труда получить оценку остаточного члена.

Каноническое произведение $V(z)$ послужит нам основой для построения функций $g_{T}(x)$. Пусть $0<\alpha<2 \pi$. Положим $V_{\alpha}(z)=V\left(z e^{-i \alpha}\right)$. Функция $V_{\alpha}(z)$ имеет своими нулями числа $\nu^{1 / e} e^{i \alpha}$. В силу равенства (3.14) для положительных $r$

$$
\begin{aligned}
\ln \left|V_{\alpha}(r)\right| & =\frac{\pi}{\sin \pi \varrho} r^{\varrho} \cos \varrho(\pi-\alpha)+O\left(r^{p}\right), \\
\ln \left|V_{\alpha}(-r)\right| & =\frac{\pi}{\sin \pi \varrho} r^{\varrho} \cos \pi \varrho \alpha+O\left(r^{p}\right) .
\end{aligned}
$$

Выбирая $\alpha=(3 \pi) /(2 \varrho)$, получим, что

$$
\ln \left|V_{\alpha}(r)\right|=-\pi r^{\rho}+O\left(r^{p}\right), \quad \ln \left|V_{\alpha}(-r)\right|=O\left(r^{p}\right) .
$$

Далее, функция $U_{\alpha}(z)=V_{\alpha}(z) V_{-\alpha}(z)$ на вещественной оси равна

$$
\prod_{r=1}^{\infty}\left|1-r e^{-i \alpha}\right|^{2} \exp \left\{\sum_{1}^{p} \frac{1}{k} \Re\left(r e^{-i \alpha}\right)^{k}\right\}
$$

и, следовательно, вещественна там. При этом $\left|V_{\alpha}(z)\right|=\left|V_{-\alpha}(z)\right|$. Положим теперь

$$
\gamma(x)=U_{\alpha}(x) U_{\alpha}(-x),
$$

это и будет искомая функция $\gamma$.

Убедимся, что она действительно обладает всеми необходимыми свойствами. Заметим сперва, что если $|z| \leqslant \delta T, \delta$ - достаточно малое положительное число, то $|\arg (T-z)| \leqslant 2 \delta$. Отсюда и из (3.14)-(3.16) следует, что для $|z| \leqslant \delta T$ выполняется неравенство $\left|\gamma_{T}(z)\right| \leqslant C_{\delta}$. В силу (3.13) для $|z|>\delta T$ выполняется неравенство $\left|\gamma_{T}(z)\right| \leqslant C e^{c_{\delta}|z|^{\ell}}$. Taким образом $\gamma_{T} \in \mathbf{F}_{1}$. Далее, из (3.16) следует, что $\sup _{|x| \leqslant 1}|\gamma(x)| \leqslant$ 
$C e^{-c T^{e}}$. Следовательно, опять информация Фишера второй вспомогательной задачи $I(\theta) \leqslant C e^{-c T^{e}}$. Теорема 1.2 доказана полностью.

4. Доказательство теоремы 1.3. Рассмотрим следующую задачу оценивания (вторая вспомогательная задача п. 3): оценить плотность распределения $f \in \mathbf{F}=\mathbf{F}_{d}(M, \sigma,(1, \ldots, 1))$ на кубе $G=[-1,1]^{d}$ по наблюдениям

$$
Y_{1}, Y_{2}, \ldots, Y_{n}, \quad Y_{j}=X_{j} I\left(X_{j}\right) .
$$

Мы уже отмечали, что если $f^{*}$ пробегает все статистики $f^{*}\left(Y_{1}, \ldots, Y_{n}\right)$, a $\hat{f}$ - все статистики $\hat{f}$, построенные по цензурированной кубом $G$ выборке (1.1), то

$$
\inf _{f^{*}} \sup _{f \in \mathbf{F}} \mathbf{E} \int_{G}\left|f^{*}(x)-f(x)\right|^{2} d x \leqslant \inf \sup _{f \in \mathbf{F}} \mathbf{E} \int_{G}|\hat{f}(x)-f(x)|^{2} d x .
$$

Поэтому нам достаточно доказать, что в задаче оценивания $f$ по выборке (4.1)

$$
\inf _{f^{*}} \sup _{f \in \mathbf{F}} \int_{G}\left|f^{*}(x)-f(x)\right|^{2} d x \geqslant c \frac{1}{n}\left(\frac{\ln n}{\ln \ln n}\right), \quad c>0 .
$$

Это последнее неравенство и доказывается ниже.

Для оценки левой части (4.3) мы воспользуемся методом из [12]; точнее его вариантом из [4]. Обозначим $\|f\|$ норму $f$ в $L_{2}(G)$. Рассмотрим некоторое конечное семейство плотностей $\left\{f_{1}, \ldots, f_{N}\right\}$ из $\mathbf{F}$ такое, что $\left\|f_{i}-f_{j}\right\| \geqslant \delta$. Тогда для любой оценки $f^{*}$

$$
\begin{aligned}
\delta^{-2} \sup _{f \in \mathbf{F}} \mathbf{E}_{f}\left\|f^{*}-f\right\|^{2} & \geqslant \delta^{-2} \sup _{f_{j}} \mathbf{E}_{f}\left\|f^{*}-f\right\|^{2} \\
& \geqslant(4 N)^{-1} \sum_{1}^{N} \mathbf{P}_{f_{j}}\left\{\left\|f^{*}-f_{j}\right\|>\frac{\delta}{2}\right\}=\frac{1}{4} p_{e} .
\end{aligned}
$$

Если $\eta$ - случайный элемент, принимающий значения $f_{j}, j=1, \ldots, N$, с вероятностями $N^{-1}$, то по лемме Фэно (см., например, [5]; ср. также [12])

$$
p_{e} \geqslant 1-(\ln N)^{-1} I\left(\eta ;\left(Y_{1}, \ldots, Y_{n}\right)\right),
$$

где через $I(\eta ; \xi)$ обозначается информация Шеннона о случайном элементе $\eta$ в случайном элементе $\xi$ (относительно количества информации по Шеннону см., например, [3]). Поскольку для независимых $\xi_{1}, \xi_{2}, \ldots, \xi_{n}$

$$
I\left(\eta ;\left(\xi_{1}, \xi_{2}, \ldots, \xi_{n}\right)\right) \leqslant \sum_{1}^{N} I\left(\eta ; \xi_{j}\right)
$$

To

$$
p_{e} \geqslant 1-n(\ln N)^{-1} I\left(\eta ; Y_{1}\right) .
$$

Оценка $I\left(\eta ; Y_{1}\right)$ осуществляется тем же способом, что и в [12]. Пусть $\mu, \nu$ обозначают меру Лебега и считающую меру, сосредоточенную на множестве целых чисел соответственно, и пусть $m=\mu+\nu$. 
Если величины $X_{j}$ имеют плотность распределения $f$, то распределения величин $Y_{j}$ имеют по отношению к $m$ плотность распределения

$$
p(x)=f(x) I(x)\left(1-I_{0}(x)\right)+I_{0}(x) \int_{\mathbf{R}^{d}} f(x)(1-I(x)) d x .
$$

Здесь $I, I_{0}$ - индикаторы $G$ и $\{0\}$ соответственно. Пусть $f_{0}$ - какаянибудь плотность распределения и пусть $p_{0}(x)=f_{0}(x) I(x)\left(1-I_{0}(x)\right)+$ $I_{0}(x) \cdot \int_{\mathbf{R}^{d}} f_{0}(x)(1-I(x)) d x$. Теми же методами, что и в [12], неравенство (3.3) этой работы, доказывается, что

$$
I\left(\eta ; Y_{1}\right) \leqslant \max _{1 \leqslant i \leqslant N} \int_{G} \frac{\left(p_{i}(x)-p_{0}(x)\right)^{2}}{p_{0}(x)} d x
$$

и, следовательно,

$$
\sup _{f \in \mathbf{F}_{\sigma}} \mathbf{E}_{f}\|\hat{f}-f\|^{2} \geqslant \frac{\delta^{2}}{4}\left(1-\frac{n}{\ln N} \max _{1 \leqslant i \leqslant N} \int_{G} \frac{\left(f_{i}(x)-f_{0}(x)\right)^{2}}{f_{0}(x)} d x\right) .
$$

Определим семейство плотностей $\mathbf{A}=\{f(x ; \mathbf{a})\}, \mathbf{a}=\left\{a_{\nu}: \nu=\right.$ $\left.\left(\nu_{1}, \ldots, \nu_{d}\right), \nu_{j}=1,2, \ldots, M\right\}$ равенством

$$
f(x ; \mathbf{a})=\prod_{1}^{d} \frac{\sin ^{2} \gamma x_{j}}{\pi \gamma x_{j}^{2}}\left(1+\prod_{j=1}^{d} \prod_{l=1}^{2 M} \frac{\sin \left(\gamma l^{-2} x_{j}\right)}{x_{j}} \sum_{\nu} a_{\nu} P_{\nu}(x)\right) .
$$

Здесь, как и в п. $2 P_{\nu}(x)=P_{\nu_{1}}\left(x_{1}\right) \cdots P_{\nu_{d}}\left(x_{d}\right), P_{\nu_{i}}\left(x_{i}\right)$ - ортонормированные полиномы Лежандра. Коэффициенты $a_{\nu}= \pm 1$, если все $\nu_{i}-$ нечетные числа, коэффициенты $a_{\nu}=0$, если хоть один из индексов $\nu_{i}$ - четное число. Общее число функций семейства $N=c_{d} M^{d}$. Ясно, что все $f(z ; \mathbf{a})$ суть целые функции конечной степени не выше $3 \gamma$ и для $\gamma \leqslant \sigma_{i} / 3, i=1, \ldots, d$, все $f \in \mathbf{F}$ с заданным $\sigma$ и некоторым $M$.

Поскольку $P_{2 k+1}(x)$ - нечетные функции,

$$
\int_{\mathbf{R}^{d}} f(x) d x=\left(\frac{1}{\pi \gamma} \int_{-\infty}^{\infty} \frac{\sin ^{2} \gamma x}{x^{2}} d x\right)^{d}=1 .
$$

Покажем теперь, что $f(x) \geqslant 0$. Имеем

$$
\prod_{j=1}^{d} \prod_{l=1}^{2 M}\left|\frac{\sin \gamma l^{-2} x_{j}}{x_{j}}\right|\left|\sum_{\nu} a_{\nu} P_{\nu}(x)\right| \leqslant h\left(x_{1}\right) \cdots h\left(x_{d}\right),
$$

где

$$
h(y)=\prod_{1}^{2 M}\left|\frac{\sin \gamma l^{-2} y}{y} \| \sum_{1}^{M}\right| P_{k}(y)|| \leqslant \min \left(\frac{1}{(2 M !)^{2}}, \frac{1}{|y|^{2 M}}\right) \sum_{1}^{M}\left|P_{k}(y)\right| .
$$

В силу (2.5) для $|y| \leqslant 1$

$$
h(y) \leqslant \frac{2 M \sqrt{M}}{(2 M !)^{2}} \leqslant \frac{1}{2},
$$


если $M>2$. В силу $(2.6)$ для $1<|y| \leqslant 4$

$$
h(y) \leqslant M \sqrt{2 M} \cdot 8^{M} \cdot(2 M !)^{-2} \leqslant \frac{1}{2},
$$

если $M>12$. Наконец, снова в силу (2.6), для $|y|>4$

$$
h(y) \leqslant M \sqrt{2 M}(|y|)^{-2 M}|y|^{M} \leqslant M \sqrt{2 M} 2^{-M} \leqslant \frac{1}{2},
$$

если $M>4$. Поэтому для всех $M>12$ выполняются неравенства

$$
f(x ; \mathbf{a}) \geqslant \frac{1}{2} \prod_{i=1}^{d} \frac{\sin ^{2} \gamma x_{i}}{\gamma \pi x_{i}^{2}}>0,
$$

и все функции семейства $\mathbf{A}$ суть плотности распределения из $\mathbf{F}$.

Семейство $\mathbf{A}$ послужит нам базой для построения семейства плотностей $f_{j}$ из неравенства (4.6). Заметим сперва, что если $|y| \leqslant 1$, $0<\gamma \leqslant 1$, то

$$
\gamma\left(1-\frac{\gamma^{2} y^{2}}{6}\right) \leqslant \frac{\sin \gamma y}{y} \leqslant \gamma\left(1-\frac{\gamma^{2} y^{2}}{8}\right) .
$$

Поэтому найдутся такие положительные постоянные $0<c \leqslant C<\infty$, что для $0<\gamma \leqslant 1$

$$
\begin{aligned}
c \gamma^{4 d M}(2 M !)^{-2 d} \sum_{\nu}\left|a_{\nu}-b_{\nu}\right|^{2} & \leqslant\|f(\cdot ; \mathbf{a})-f(\cdot ; \mathbf{b})\|^{2} \\
& \leqslant C \gamma^{4 d M}(2 M !)^{-2 d} \sum_{\nu}\left|a_{\nu}-b_{\nu}\right|^{2} .
\end{aligned}
$$

Лемма 4.1. Пусть фуниции $\mathbf{a}(\omega)$ определены на хонеином множестве $\Omega, \operatorname{card} \Omega=L$, и принимают там значения \pm 1 . Найдется такое множество $\mathbf{A}_{0}$ бункиий $\mathbf{a}$, ито $\sum_{\omega}\left|\mathbf{a}(\omega)-\mathbf{a}^{\prime}(\omega)\right| \geqslant L / 4$ для всех пар $\mathbf{a}, \mathbf{a}^{\prime}$ из $\mathbf{A}_{0} u$

$$
\operatorname{card} \mathbf{A}_{0} \geqslant\left(2^{-L} \sum_{k=0}^{[L / 4]} C_{L}^{k}\right)^{-1} \geqslant \exp \left\{\frac{L}{8}\right\} .
$$

Первое из неравенств (4.8) - это неравенство Гилберта или ВаршамоваГилберта из теории кодирования, см. [2, с. 546-547], второе неравенство легко следует из элементарных теорем о вероятностях больших уклонений для испытаний Бернулли. Подробнее см. [6].

Из леммы 4.1 и неравенства (4.7) следует, что из семейства плотностей $\mathbf{A}$ можно выделить такое подмножество $\mathbf{A}_{0} \subset \mathbf{A}$, что саrd $\mathbf{A}_{0} \geqslant$ $C \exp \left\{c M^{d}\right\}, C, c>0$ и что

$$
\delta^{2}=\min \left\|f_{i}-f_{j}\right\|^{2} \geqslant c_{0} \gamma^{4 d M}(2 M !)^{-2 d} M^{d}, \quad c_{0}>0,
$$


где минимум берется по всем $f_{i}, f_{j}, i \neq j$ из $\mathbf{A}_{0}$. Примем за $\mathbf{A}_{0}$ семейство плотностей $f_{i}, i=1, \ldots, N, N \geqslant C e^{c M^{d}}$, из неравенства (4.6). Положим

$$
f_{0}(x)=\prod_{j=1}^{d} \frac{\sin ^{2} \gamma x_{j}}{\pi \gamma x_{j}^{2}}
$$

Из неравенств (4.9) и (4.6) следует тогда, что для любой оценки $f^{*}$

$$
\sup _{f \in \mathbf{F}} \mathbf{E}_{f}\left\|f^{*}-f\right\|^{2} \geqslant \frac{\delta^{2}}{4}\left(1-C \cdot n(\ln N)^{-1} \cdot \gamma^{4 M d}(2 M !)^{-2 d} M^{d}\right) .
$$

Выберем в качестве $M$ наименьшее целое число, для которого $C \gamma^{4 M d}(2 M !)^{-2 d} M^{d} \cdot(\ln N)^{-1} \cdot n \leqslant \frac{1}{2}$. Тогда $M \asymp \ln n / \ln \ln n$ и для всех больших $n$

$$
\sup _{f \in \mathbf{F}} \mathbf{E}_{f}\left\|f^{*}-f\right\|^{2} \geqslant \frac{\delta^{2}}{16} \geqslant \frac{c}{n}\left(\frac{\ln n}{\ln \ln n}\right)^{d} .
$$

Последнее неравенство доставляет нам нужную нижнюю границу и вместе с теоремой 2.1 доказывает теорему 1.3 .

\section{СПИСОК ЛИТЕРАТУРЫ}

1. Бернштейн C. Н. Экстремальные свойства полиномов и наилучшее приближение непрерывныг функций вещественной переменной. М.-Л.: ОНТИ, 1937, 203 с.

2. Галлагер $P$. Теория информации и надежная связь. М: Советское радио, 1974, $720 \mathrm{c}$.

3. Гельфанд И. М., Яглом А. М. О вычислении количества информации о случайной функции, содержащейся в другой такой функции. - Успехи матем. наук, 1957, т. 12 , № 1 , c. $3-52$.

4. Ибрагимов И.А. Об оценивании аналитической плотности распределения по цензурированной выборке. - Зап. научн. семинаров ЛОМИ, 2004, т. 311, с. 147-160.

5. Ибрагимов И. А., Хасминский Р. З. Асимптотическая теория оценивания. М.: Наука, 1979, 527 с.

6. Ибрагимов И. А., Хасминский Р. З. Об оценке плотности распределения. - Зап. научн. семинаров ЛОМИ, 1980, т. 98 , с. 61-85.

7. Ибрагимов И. А., Хасминский Р. З. Об оценке плотности распределения, принадлежашей одному классу целых функций. - Теория вероятн. и ее примен., 1982, т. XXVII, в. 3 , с. $514-524$.

8. Ибрагимов И. А. Об экстраполяции целых функций наблюдаемых в гауссовском белом шуме. - Укр. матем. журнал, 2000, т. 21, в. 3, с. 58-69.

9. Левин Б. Я. Распределение корней целых функций. М.: Гостехиздат, 1956, 632 с.

10. Сеге Г. Ортогональные многочлены. М.: Физматгиз, 1962, 500 с.

11. Стейн И., Вейс Г. Введение в гармонический анализ на евклидовых пространствах. М.: Мир, 1974, 331 с.

12. Hasminskii R., Ibragimov I. On density estimation in the view of Kolmogorov's ideas in approximation theory. - Ann. Statist., 1990, v. 18, № 3, p. 999-1010.

13. Хермандер Л. Введение в теорию функций нескольких комплексных переменных. М.: Мир, 1968, 279 с.

14. Ченцов $H$. $H$. Оценка неизвестной плотности распределения по наблюдениям. Докл. АН СССР, 1962, т. 147, № 1, с. 45-48. 